\title{
ISOLEMENT DES RÉSONANCES D'UNE PLAQUE METALLIQUE RECOUVERTE PAR UN POLYMÈRE
}

J.L. IZBICKI, O. LENOIR, P. REMBERT, P. PAREIGE, J.L. ZUBER, G. MAZE et J . RIPOCHE

\author{
Laboratoire d'Acoustique Ultrasonore et d'Électronique, CNRS \\ URA-1373, Université du Havre, Place Robert Schuman, F-76610 Le Havre, \\ France
}

\begin{abstract}
Resume - Les résonances d'une plaque solide metallique contenue dans des structures du type polymere/couplant/plaque metallique sont étudiees. Les structures sont insonees par une impulsion breve; le signal reflechi est traite par un algorithme de TFD. Si le polymere n'est pas trop absorbant, des spectres de resonances et des spectres provenant de la remission des ondes guidees permettent d'isoler les résonances de la plaque métallique. Si le polymère est absorbant, (sa présence provoque une atténuation d'au moins $15 \mathrm{~dB}$ sur I'amplitude du premier echo par rapport a la reflexion sur la plaque metallique seule) une méthode mettant en jeu la phase de la totalite du signal reflechi permet l'isolement des resonances de la plaque metallique.
\end{abstract}

Abstract - The resonances of a metallic plate included in polymer/coupling/metallic plate are studied. in all the cases, short pulse excitation is used; the reflected signal is processed by a FFT algorithm. If the polymer is not too absorbant, resonance spectra or spectra obtained from the guided wave reemission allow the isolation of the metallic plate resonances. If the polymer is absorbant (Its presence induces a $15 \mathrm{~dB}$ attenuation on the amplitude of the first echo comparatively to the reflection of the metalilo plate alone), the study of the phase of the whole reflected signal provides the resonance frequencies of the metallic plate.

\section{INTRODUCTION.}

\begin{abstract}
Le caractere resonnant de la diffusion acoustique par des couches elastiques planes est etabli theoriquement depuis longtemps [1]. Experimentalement, l'etude de la reflexion d'une onde incidente sur une plaque elastique met en évidence ce caractere résonnant. Lorsque I'onde incidente est constituee par un train de sinusoides suffisamment long, afin quiun regime permanent de vibration soit etabli dans la plaque, une extension de la methode MIIR [2] a permis 1'isolement des resonances de la plaque [3]. Lorsque l'excitation inoidente est réalisee par une $1 \mathrm{mpulsion}$ brève, il est également possible d'obtenir le spectre des resonances de la plaque en analysant une partie du signal reflechl par la plaque [4-5].

Des experiences de diffusion acoustique permettant l'lsalement des résonances d'une plaque metallique recouverte par un polymere sont rapportées ici. Dans une premiere partie, une plaque plastique recouvre une plaque en aluminium; le couplage entre les deux plaques est assure par un mince film d'eau. Dans une seconde partie, des expériences concernant une couche d'absorbant collée sur une plaque d'aluminium sont decrites. Dans tous les cas de figure, une znéthode de detection des resonances de la couche d'aluminium est proposée. Au préalable, les conditions
\end{abstract} experimentales sont brievement décrites. 


\section{PROCEDURES EXPER I MENTALES}

Les transducteurs et les multicouches sant immergés dans une cuve remplie. d'eau. Les plaques utilisees ont des dimenstons telles qu'il n'y a pas d'ondes stationnaires qui s'etablissent dans la longueur ou la largeur des plaques. La zone d'insonation est toujours tres inferieure a la surface totale de la structure etudiee. Une impulsion brève est appliquee a un transducteur large bande. Dans la methode monostatique, le transducteur fonctionne egalement en recepteur; 11 insone la structure multicouche sous incidence normale. En fait, lorsque l'on parle d'incidence normale, il s'agit de la direction moyenne du faisceau incident qui est normale a la structure plane : une partie de l'energie incidente arrive sur la structure sous incidence oblique a cause de la dimension finie de la pastille d'émission. Une partie de l'energie reçue correspond a une reflexion sous incidence oblique; les niveaux d'amplitude correspondant sont cependant faibles vis a vis du niveau correspondant a l'incidence normale. Ceci explique pourqual, sous "incidence normale", il est possible, experimentalement, d'exciter des ondes transversales dans un solide. Dans la méthode bistatique, un deuxieme transducteur, identique au transducteur emetteur, fonctionne en recepteur. Ce transducteur recepteur peut etre positionné soit en position "Sneli-Descartes" - il s'agit alors d'etudier la réflexion spéculaire du faisceau incident -, soit en position "guidee". Dans ce cas, le transducteur, positionne sous un angle egal a l'angle de reflexion, est decale parallelement au plan de la plaque par rapport au transducteur emetteur [6]: il s'agit d'etudier la rémission d'ondes guidees se propageant sur la structure et rémettant dans le liquide sous un angle correspondant à l'angle d'excitation.

Une transformée de Fourier discrete (TFD) est réalisée sur tout ou partie du signal reçu. On obtient, pour chaque frequence, un nombre complexe. I1 est usuel d'etudier le module de ce nombre complexe cou le logarithme du module) en fonction de la frequence : on obtient un spectre de puissance analogue à celui obtenu en methode quasi-harmonique et appele spectre de retrodifiusion - tout le signal reçu est traite - et des spectres de résonances - seule la partie correspondant a la rémission libre de la structure est traitee apres filtrage temporel. Il est montre qu'il est interessant egalement d'examiner la partie reelie et la partie imaginaire du nombre complexe calcule par TFD [7-8]. Tous les spectres presentes dans la suite présentent une echelle logarithmique en ordonnee; les spectres ne sont pas corrigés par la prise en compte de la réponse en fréquence des transducteurs.

\section{ETUDE DE LA STRUCTURE MAKROLON/EAU/ALUMI IUM}

Il s'agit d'etudier une structure multicouche plane makrolon/eau/alumintum (structure "MEA") dans laquelle le makrolon est une couche plastique; l'eau "interne" sert de couplant entre les deux couches solides. Les epaisseurs des couches sont respectivement $4 \mathrm{~mm}, 1 \mathrm{~mm}$ et $2 \mathrm{~mm}$. Une étude de la plaque de makrolon a montre que celle-ci ne présente pas de régime de rémission libre, $n i$ en incidence normale, ni en incidence oblique [8], Le signal retrodiffuse par la structure obtenu par la methode monostatique est compose de trois parties notees $A, B, C$ (Fig. 1 ). La partie A correspond aux réflexions sur la premiere interface eau-makrolon et sur la deuxieme interface makrolon-eau (interne); la partie B comporte a la fois des reflexions et des transmissions dans la structure; la partie $C$ correspond a la reemission libre de la multicouche. Le spectre de la partie A (Fig. 2), le reste du signal ayant eté filtré temporellement, correspond a celui obtenu à partir de la plaque de makrolon considerée isalement : les creux correspondent a l'excitation d'ondes longitudinales a l'interieur de cotto coiche. Le spectre de la totalite du signal retrodiffuse montre (Fig. 3) essentieliement des variations amples qui ne sont pas caracteristiques de la plaque d'aluminium mais plutot de la plaque de makralon. Le spectre 
de la partie C (Fig. 4) montre un pio large à $1,56 \mathrm{MHz}$ lie aux modes de Lamb $S 1$ et $S 2$ de l'aluminium : cette frequence est celle qui est obtenue Iors de l'etude d'une plaque d'alumintum consideree isolement. On ne detecte pas de resonances liees au makralon ou a la couche d'eau interne; la presence d'une plaque de makrolon devant la plaque d'aluminium n'influe pas sur la postion frequentielle de la résonance. Une etude des ondes guidees paralielement au plan de la structure permet egalement la detection des resonances de la plaque d'aluminium. Pour un angle dincidence de $5^{\circ}$, le signal provenant de la rémission des ondes guidées (Fig. 5A) présente des paquets d'onde successifs dont la forme en losange est caracteristique de la propagation d'andes guidés dans l'aluminium. Le spectre de puissance de ces paquets d'ondes (Fig. 5B) permet l'lsolement des pics de résonances aे $850 \mathrm{kHz}, 1,66 \mathrm{MHz}, 3,34 \mathrm{MHz}$ qui correspondent aux modes de Lamb A1, S2, A3 de I'aluminium.

\section{ETUDE DE LA STRUCTURE POLYSTYREHE/EAU/ALUMIMIUH}

La multicouche etudiee est composée d'une plaque de polystyrene de $2 \mathrm{~mm}$ d'épaisseur, d'une couche d'eau d'environ $0,2 \mathrm{~mm}$ d'epaisseur et d'une plaque d'aluminium de $5 \mathrm{~mm}$ d'épaisseur (structure "PEA"). Contratrement a la plaque de makrolon, le spectre de la reponse élastique de la plaque de polystyrene présente des pics de resonance. La figure 6 represente le spectre des résonances obtenu par la methode monostatique de la plaque de polystyrene seule; l'obtention de ce spectre est difficile a cause d'un mauvals rapport signal sur bruit. Deux pics de résonance à $580 \mathrm{kHz}$ (mode 52 ) et $1,72 \mathrm{MHz}$ (mode 55 ) sont lies a des ondes longitudinales; les deux autres situes a $860 \mathrm{kHz}$ (mode. A2) et $1,42 \mathrm{MHz}$ (mode A4) sont $11 \in$ ) a des ondes transversales dans la plaque. En incidence oblique, les resonances sont plus faciles a obtentr et sont plus nombreuses (F18. 7). Cette plaque plastique presente donc un caractère résonnant. Le probleme qui se pose est le suivant : peut-on separer experimentalement les contributions résonnantes de chacune des plaques resonnantes de la structure PEA?

En incidence normale, le signal reflechi par la multicouche (Fig. 8 ) comprend une premiere partie notee A composee de 2 echos de refiexion aux interfaces eau/polystyrene ou polystyrene/eau (interne), une seconde partie notee $B$ comprenant une succession de reflexions et de transmissions dans chaque couche de la multcouche et une traisieme partie notee $C$ correspondant a la reponse élastique de la multicouche. Le spectre de puissance de la totalite du signal rétrodiffuse (Fig, 9 ) a la forme generale du spectre de rétrodiffusion de la-plaque d'alumintum seule : les minima observes a $0,62 \quad 0,66 \quad 1,30 \quad 1,90 \quad 1,96 \quad 2,62 \quad 3,28 \mathrm{MHz}$ peuvent etre observes sur le spectre de rérodiffusion de la plaque d'aluminium de 5 mm etudiee isolement [8]. On note par allieurs des oscillations d'amplitude faible comparee aux precedentes qu'il n'est pas possible d'attribuer a I'une ou l'autre des plaques solides. Elles ne sont pas liees non plus a des phenomenes résonnant dans la couche d'eau : son épaisseur est trop faible pour observer des resonances de la couche d'eau dans la gamme de fréquence utilisée. Le spectre de la reponse elastique (Fig. 10) est essentiellement domine par des ptos de resonances lies a des modes de vibration dans l'aluminium. Les pics de resonances correspondant a la réponse elastique du polystyrene ne sont pas aisement isolabies : en incidence normale la dynamique des pics dus a l'aluminium est beaucoup plus importante que celle des pios dus au polystyrene. En inoidence oblique, le spectre du régime libre de la structure PEA ne permet pas la separation des contributions de chaque plaque et en particulier la detection claire des résonances de la plaque metaliique. Afin de séparer les contributions resonnantes de chaque plaque, une etude de. la propagation d'ondes guidees dans la multicouche est réalisee. L'angle d'incidence est fixe $25^{\circ} ; l^{\prime}$ recepteur est placé a differentes distances de l'emetteur. Dans tous les cas, le signal observe (Fig. 12) presente une queue d'amplitude décroissante caracteristique de la propagation d'ondes guidees dans le 
polystyrène et d'un paquet d'onde en forme de losange caracteristique de la propagation d'ondes guidees dans l'aluminium. Pour une distance de $28 \mathrm{~cm}$ de propagation sur la plaque, le spectre obtenu (Fig. 13) montre un grand nombre de pics bien isoles; ils sont d'ailleurs beaucoup mieux isolés que dans le spectre des résonances obtenu avec un transducteur recepteur placé en position "Snell-Descartes". Tous les pics observés correspondent a des modes de Lamb de l'aluminium. Pour une distance de $15 \mathrm{~cm}$ de propagation le spectre obtenu (Fig. 14) montre, d'une part, les pics précedents et, d'autre part, une autre famille de pics : ceux-cl sont liés aux modes de Lamb du polystyrene. L'etude des ondes guidees permet donc bien de separer les contributions resonnantes. Bien que les deux solides soient couplés acoustiquement, les fréquences des resonances de la structure multicouche semblent etre la juxtaposition des frequences des resonances de chaque couche. Autrement dit, le couplage entre les deux plaques solides est suffisament important pour exciter des resonances de l'aluminium mals n'est pas suffisant pour modifier les frequences des résonances. Les ondes de Lamb assoctees a chaque couche semblent donc plégees dans chaque couche.

\section{ETUDE DE LA STRUCTURE ABSORBAHT/ALUMIHIUY}

Il s'agit d'etudier une multicouche comportant une couche viscoelastique de $1 \mathrm{~cm}$ d'épalsseur collee sur une plaque d'alumintum de $8 \mathrm{~mm}$ d'epaisseur. Certains resultats concernant cette structure ont eté donnes par allieurs [9]. Seules les conolusions obtenues sont rapportees ici. Pour une structure appelee ABS2/Aluminium il est possible d'obtenir un spectre des résonances mettant en evidence des resonances de la plaque d'aluminium obtenu dans les memes conditions que precedemment, et ceci bien qu'on ait affaire a un "vrał" absorbant en ce sens que le premier echo reçu dans la méthode monostatique a une amplitude diminuee de 20 dB enviran par rapport a l'amplitude du premier echo rétrodiffuse. par la plaque d'alumintum non recouverte. Ainsi, puisque le probleme pose est celui de l'isolement des resonances, 11 ne suffit pas q'un absorbant, colle sur la plaque d'aluminium, atténue le premier echo pour empecher la détection des résonances. L'energie transmise a travers la plaque d'absorbant arrive jusqu'a la plaque d'aluminium; celle-ci rentre alors en resonance pour certaines frequences. Par contre, pour la structure ABS1/Aluminium, il n'est pas possible d'observer un spectre des résonances bien que l'atténuation de l'amplitude du premier écho ne soit que de $15 \mathrm{~dB}$ environ. Une partie de l'energie transmise a travers l'absorbant est sans doute diffusee lateralement dans l'absorbant et le signal retrodiffusé n'a pas une amplitude suffisante pour pouvoir obtenir un spectre de puissance dans de bonnes conditions.

Jusqu'a maintenant, la plupart des experimentateurs se sont interesses au spectre de puissance des signaux provenant de differents objets immergés dans l'eau. Cependant le calcul de la TFD d'un signal fourntt, pour chaque fréquence, un nombre complexe. Pour obtenir les spectres "habituels", c'est à dire le spectre de retrodiffusion et le spectre des résonances, 11 suffit de s'interesser au module de ce nombre complexe. Quelques articles traitant de I'utilisation de la phase ont deja été publiés [10]. II est possible d'obtentr, à partir de l'oscilloscope numerique utilisé, l'evolution, en fonction de la frequence du rapport de la partie imaginaire a la partie reelle (note Im/Re) du nombre complexe obtenu a partir de la TFD de l'ensemble du signal reçu. Les transitions liees aux resonances se traduisent par des passages de la phase de $-\pi / 2$ à $+\pi / 2$. Donc, au voisinage d'une resonance, on peut s'attendre a observer de grandes variations du rapport Im/Re. Dans un premier temps, une plaque de PVC est utilisee. La figure 15 présente le spectre de puissance du signal rétrodiffusé par la plaque en incidence normale. Ce spectre présente l'allure classique d'un coefficlent de reflexion, les minima etant lies a l'excitation d'ondes longitudinales dans le materiau. Il s'agit bien d'une plaque absorbante car le spectre de puissance du signal transmis ne 
presente pas de maxima lies aux minima du spectre du signal reflechi. Le spectre des resonances de cette plaque n'a pas pu etre obtenu car la plaque ne presente pas de régime de rémission libre. Le spectre Im/Re (Fig. 16) fournit des informations sur l'excitation d'ondes longitudinales (L) et transversales (T) a l'interteur de la plaque. II semble donc que ce traitement apporte sensiblement plus d'informations que l'etude des spectres "habituels" dans le cas de materiau possedant des proprietes absorbantes marquees. Dans un second temps, le spectre Im/Re de Ia structure ABSi/Aluminium etudiee en incidence normale a ete trace (Fig. 17). On observe des transitions pour des frequences egales aux frequences de resonance de la plaque d'aluminium. Il semble dono interessant de considerer des grandeurs liees a la phase et pas seulement aux spectres de puissance des signaux provenant de structures présentant des proprietés viscoélastiques.

\section{CORCLUSIOH}

L'isolement des résonances d'une couche solide metallique placee derriere une couche plastique plus ou moins absorbante a fait l'objet de diverses experiences. Ces experiences mettent en jeu des traitements assez simples de signaux impulsionnels reflechis par une structure plane. Ces traitements consistent, sulvant les cas, en l'obtention des spectres de pulssance de tout ou partie du signal reçu - o'est le cas d'une structure plaque non resonnante peu absorbante/couplant (eau)/plaque metalilque au plaque peu absorbante résonnante/couplant (eau)/plaque metaliqque - en l'obtention du spectre Im/Re de tout le signal rétrodiffusé dans le cas d'une structure absorbant/couplant (colie)/plaque metalifque. Des experiences sur des objets de geometrie cylindrique sont en cours [11]; l'influence de I'absorption sur les coefficients de reflexion et de transmission par des structures planes font egalement l'objet d'etudes [12].

Une partie de ce travail est soutenue par la DRET (Contrat 88/245) que nous remercions.

\section{BIBLIOGRAPHIE}

[1] Voir $\approx .8$.

R. FIORITO, W. MADIGOSKY, H. UBERALL, Resonance theory of acoustic waves interacting with an elastio plate, J. Acoust. Soc. An., 66, 1857-1866 (1977)

[2] G. MAZE, J. RIPOCHE, Methode d'Isolement et d'Identification des Résonances (MIIR) de cylindres et de tubes soumis a une onde acoustique plane dans I'eau, Rev. Phys. Appl., 18, 319-326 (1983)

[3] J.I. IZBICKI, G. MAZE, J. RIPOCHE, Etude de la rémission libre d'une plaque résonnante, Acustica, 55, 27 (1984)

[4] M. de BILIY, Determination of the resonance spectrum of elastio bodies via the use of short pulses and Fourier transform theory, J. Acoust. Soc. Am., $79,219-221$ (1986)

[5] P. DELESTRE, J.L. IZBICKI, G. MAZE, J. RIPOCHE, Excitation acoustique impulsionnelle d'une plaque elastique immergée : application a l'isolement des résonances, Acustica, 61, 83-85 (1986)

[6] G. MAZE, J.L. IZBICKI, J. RIPOCHE, Resonances of plates and cylinders : guided waves, J. Acoust. Soc. Am., 77, 1352-1357 (1985)

[.7] P. REMBERT, O. LENOIR, J.L. IZBICKI, G. MAZE, Experimental analySis of phase spectrum of cylindrical and plane targets: a new global method of isolation of resonances, Phys. Lett., 143, 467-471 (1990)

[8] O. LENOIR, Diffusion acoustique impulsionnelle par des multicauches planes immergees. Probleme inverse. Detection des résonances en présence d'absorbant, These de Doctorat de l'Universite du Havre (1990) 
[9] . LENOIR, P. REMBERT, J.L. IZBICKI, G. MAZE, J. RIPOCHE, InfIuenCe d'absorbants sur la diffusion ultrasonore par une plaque elastique, 1 CFA Iyon 10-13 Avril 1990, J. Phys. 51C2,455-458 (1990)

[ 10$]$ Voir e.g.

N.F. HAINES, J.C. BELL, P.J. MC INTYRE, The application of broadband ultrasonic spectroscopy to the study of layered media, J. Acoust. Soc. Am., $64,1646-1651$ (1978)

N. MERCIER, J.F.. de BELLEVAL, Evaluation de I'amplitude et de la phase en analyse spectrale du signal echographique, Traitement du Signal, $5,425-433$ (1985)

[11] P. REMBERT, O. LENOIR, P. PAREIGE, J.L. IZBICKI, G. MAZE, J, RIPOCHE, Isolation of resonances of cylindrical targets : comparison between different experimental methods (MIIR, Short Pulse MIIR and Im/Re), 119 Meeting of the Acoustical Society of America, State College, Pennsylvania, USA, 22-25 mai 1990 accepte

[12] O. LENOIR, P. REMBERT, J.L. IZBICKI, P. PAREIGE, A new method for the detection of viscoelastic solid resonances : the Im/Re spectrum, Symposium on Physical Acoustics, K. U. Leuven, Campus Kortrifk (1990) accepte.
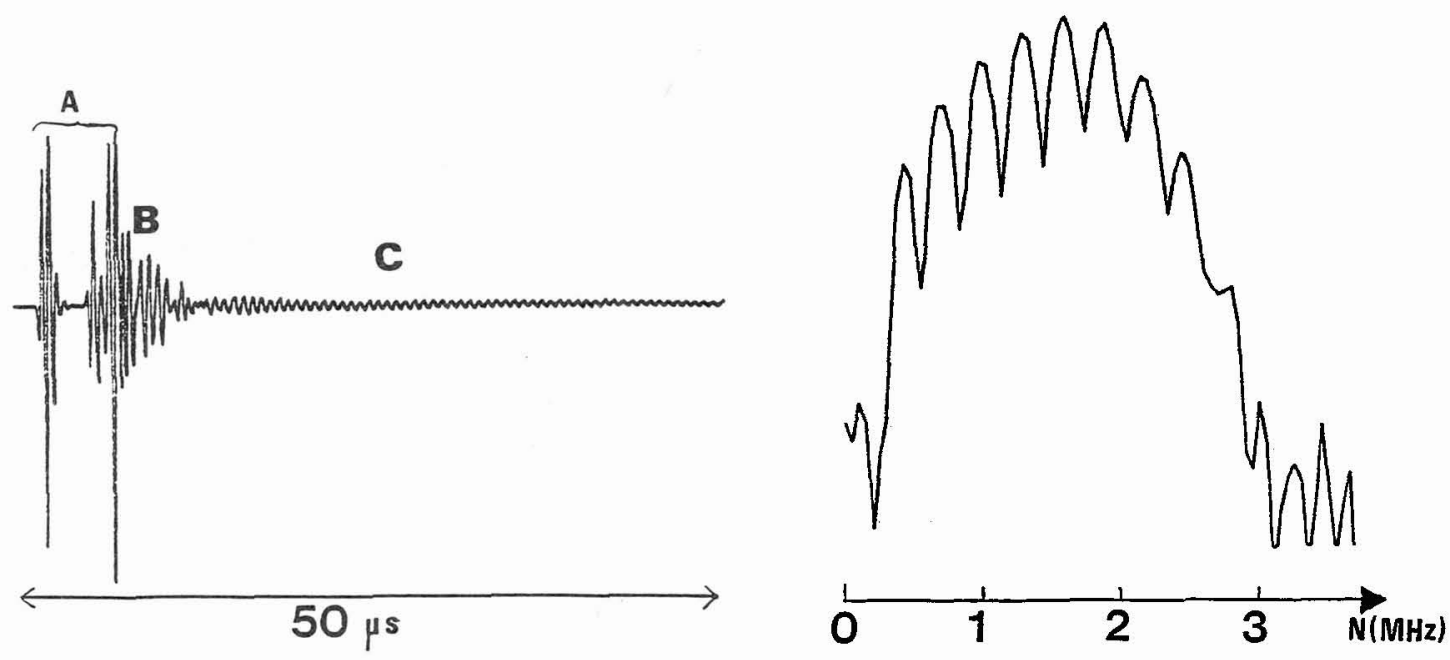

Fig. 1 Signal rétrodiffuse par la structure MEA

Fig. 2 Spectre de la partie A de la figure 1 


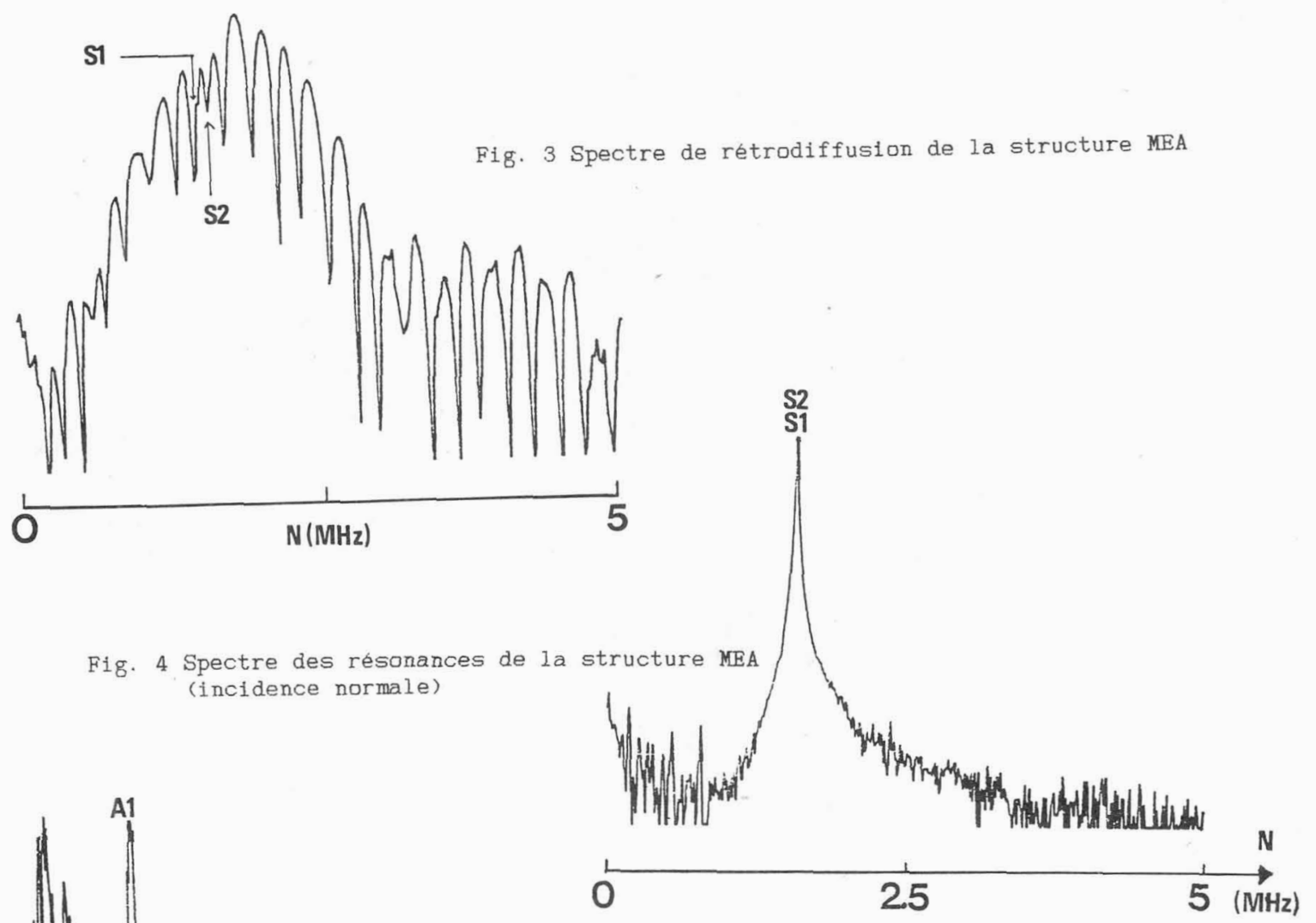

\section{S2}

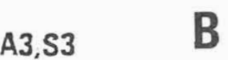

Fig. 5A Signal réémis par la structure MEA

Ondes guidees (angle d'incidence $5^{\circ}$ )

$5 B$ spectre de la figure $5 \mathrm{~A}$

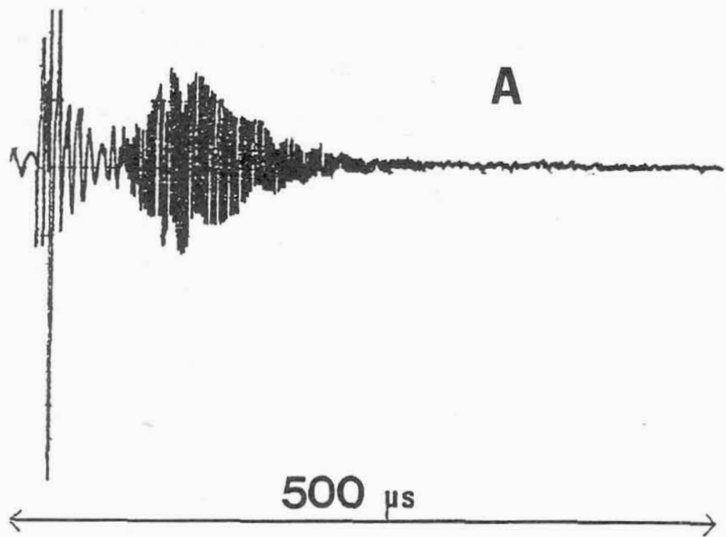



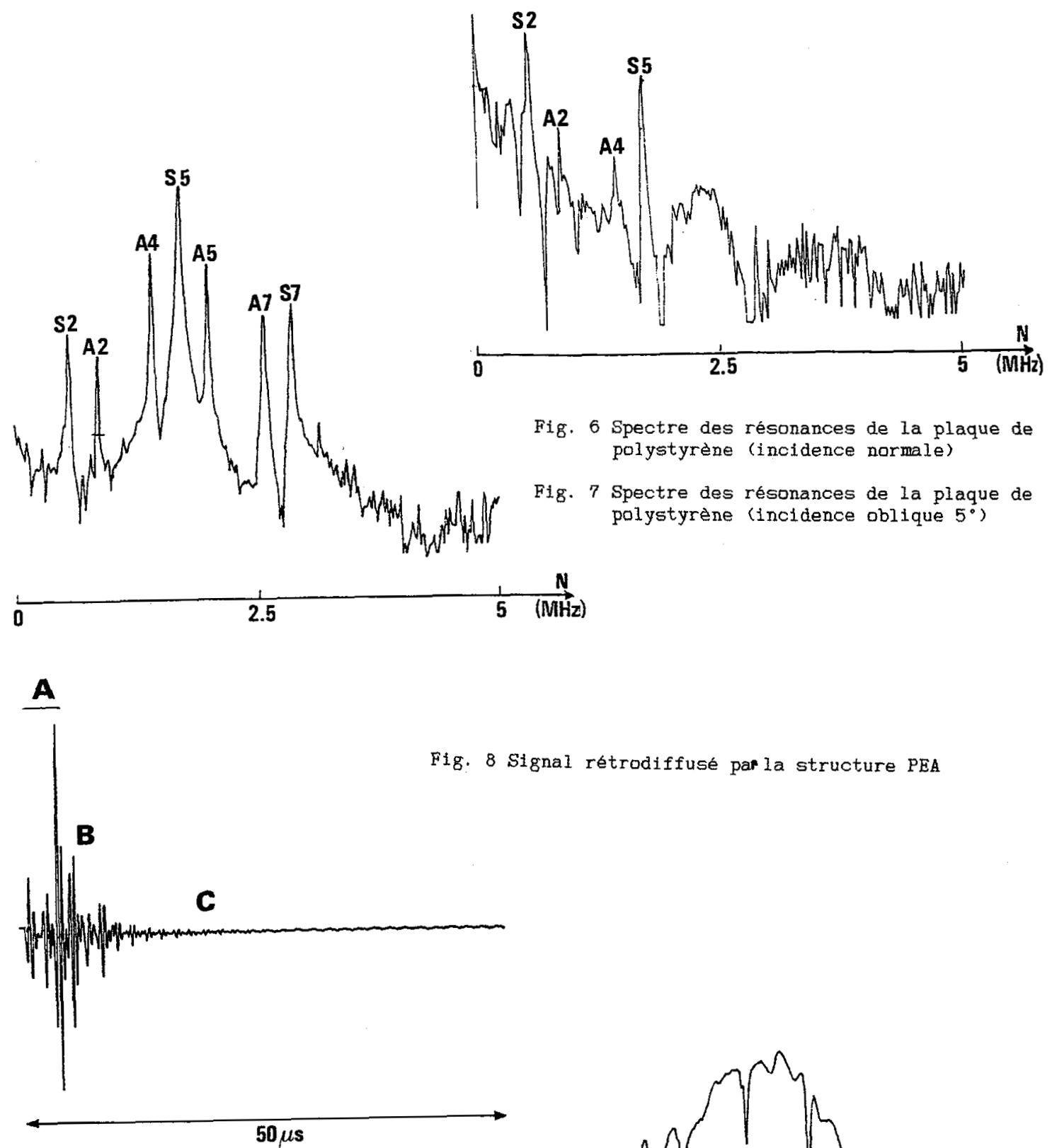

Fig. 9 Spectre de rétrodiffuston de la structure PEA

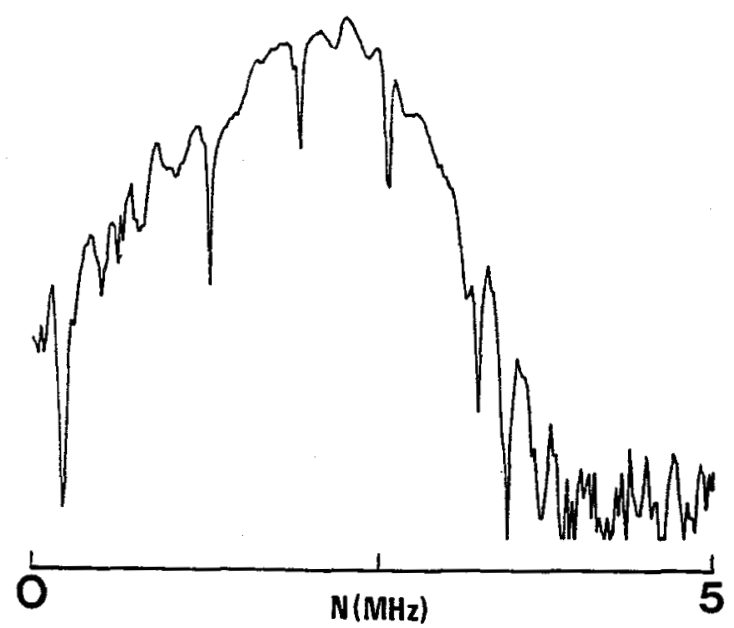


Fig. 10 Spectre des résonances

de la structure PEA

en incidence normale

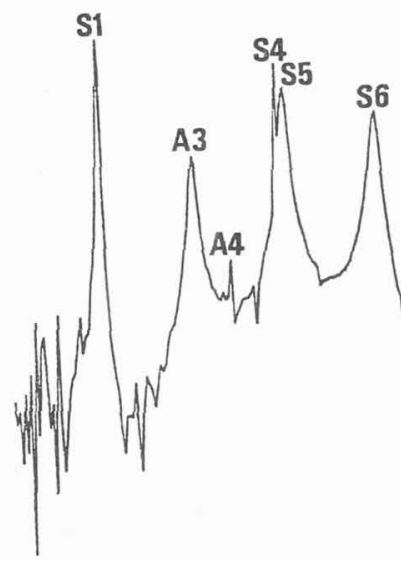

$4 \int^{58}$
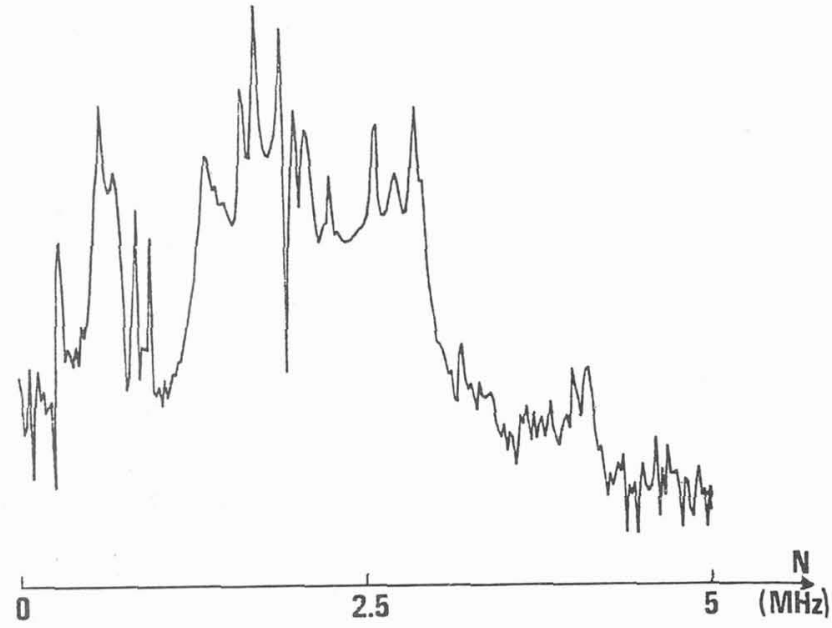

Fig. 11 Spectre des résonances

de la structure PEA

en incidence oblique $\left(5^{\circ}\right)$
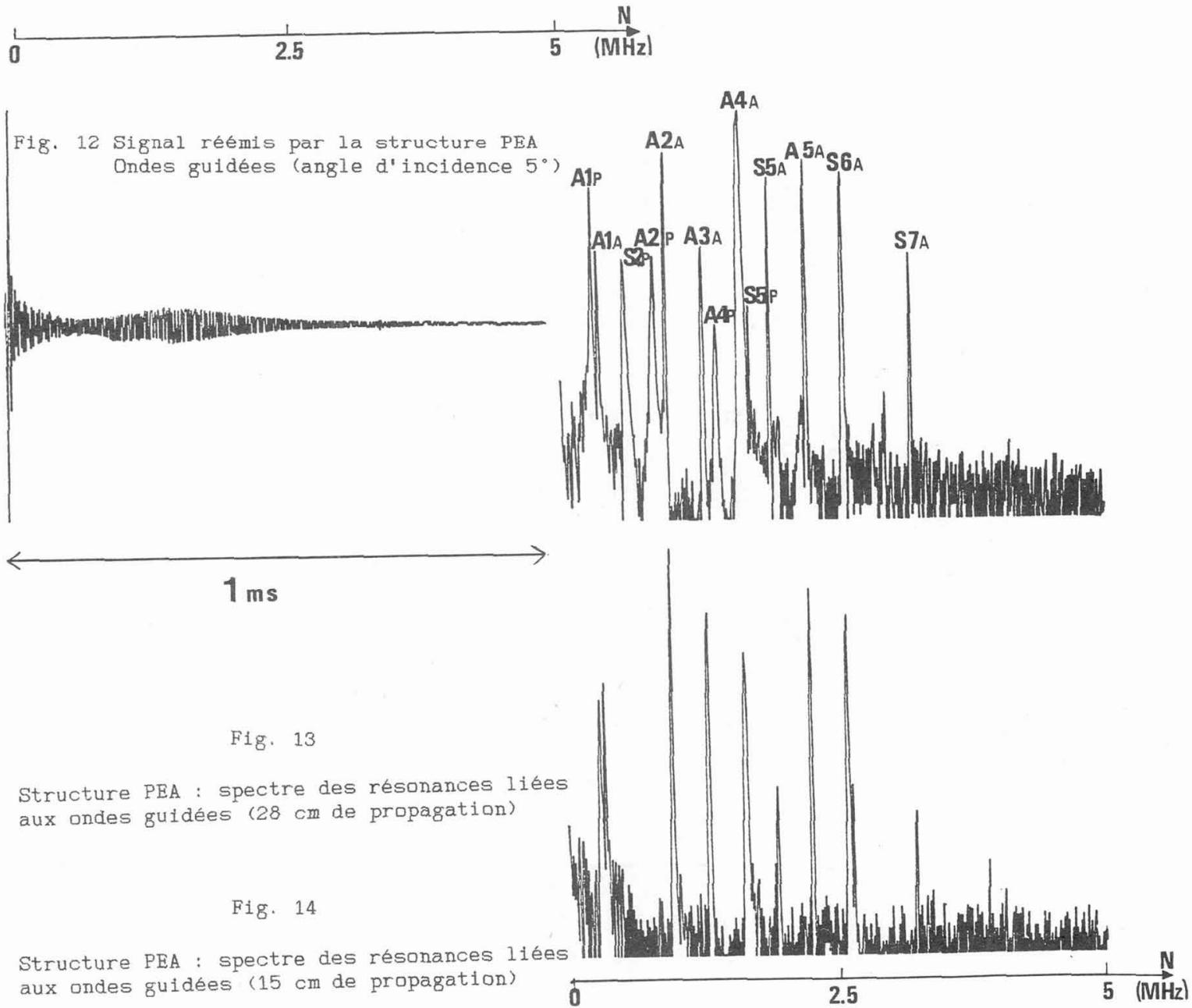


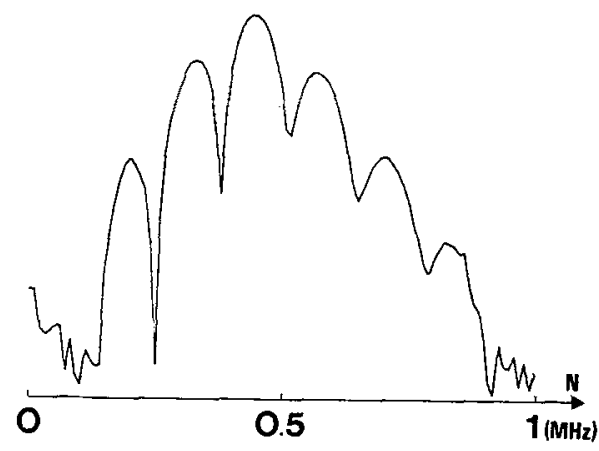

Fig. 15 Spectre de retrodiffuston de la plaque de PVC

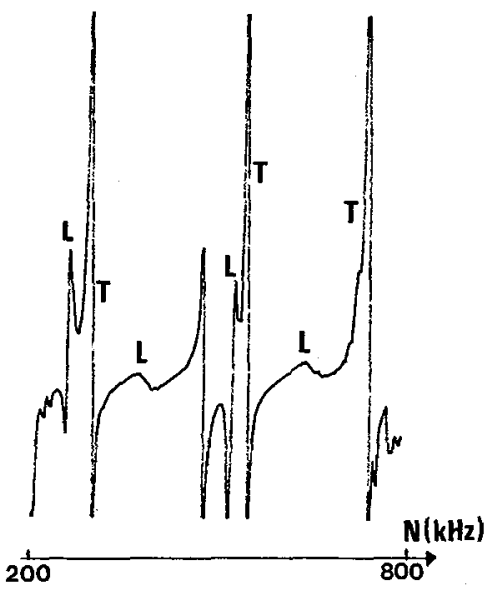

F1g. 16 Plaque de PVC : spectre Im/Re
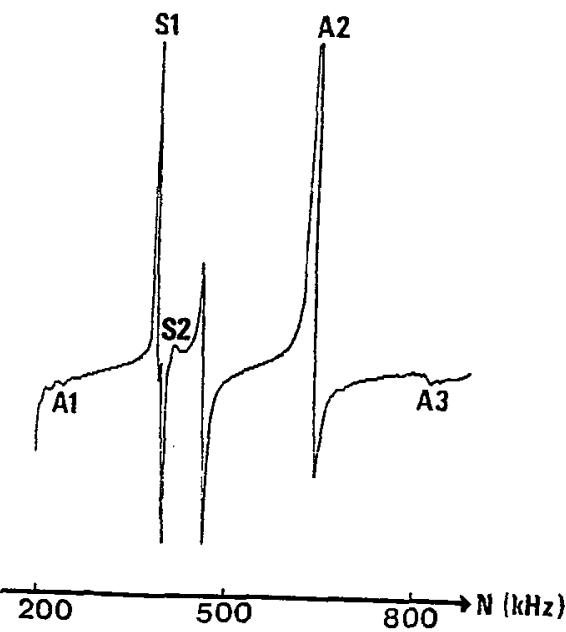

Fig. 17 Structure ABSi/Aluminium : spectre In/Re 